\title{
Measuring resistance, recovery and resilience in long-term ecological datasets
}

\author{
Alistair Seddon', L. Cole' ${ }^{2,3}$, M. Fletcher ${ }^{4}$, J. Morris ${ }^{5}$, K. Willis ${ }^{6,2}$ and EcoRe3 working group \\ EcoRe3 workshop, Finse, Norway, 27-31 March 2017
}

\begin{abstract}
The aim of the first EcoRe3 working group workshop was to develop novel, quantitative approaches to measure components of ecosystem resilience (e.g. resistance - the amount of change following a disturbance; recovery rates - the speed of recovery following disturbance; and latitude - the distance to an ecological threshold) using proxy data from lake and bog sediments. It was attended by 23 participants from 11 countries, and was split into three main thematic sessions. The workshop also included a meeting to discuss working group organization over the next three years. It was co-funded by PAGES and the Research Council of Norway.
\end{abstract}

Session one built on preliminary work which used a variety of statistical approaches to measure resistance and recovery rates in pollen data. A key issue highlighted in discussions was that the timing and the impact of disturbance events are represented by a small number of data points in many Holocene sediment sequences. Numerical techniques which can identify signal from noise are therefore crucial for quantifying any disturbance-related ecological change in such records.

Two new projects were established which aim to address this issue using different approaches. First, a systematic review will be used to investigate current knowledge about the timing and magnitude of ecological disturbances in different systems. The result of this work will lead to a series of recommendations that highlight the appropriate analyses and ecological questions for any given paleodataset. Second, a series of model-based, statistical approaches were developed to enable robust detection and quantification of any disturbance-related response. Members of EcoRe3 are now working to implement these model-based approaches in a range of ecological settings (Fig. 1)

Since a number of workshop participants were experts in measuring components of re silience using remote sensing data, a second session was dedicated to exploring methods of measuring biome stability, determining the number and presence of alternative states, and identifying distances to thresholds using a combination of both spatial and temporal datasets. A novel statistical approach was presented to address these aims using satellite data, and breakout discussion groups were held in which this method was subjected to rigorous critique and review. Discussions were then held to determine pathways for translating the approach, currently developed

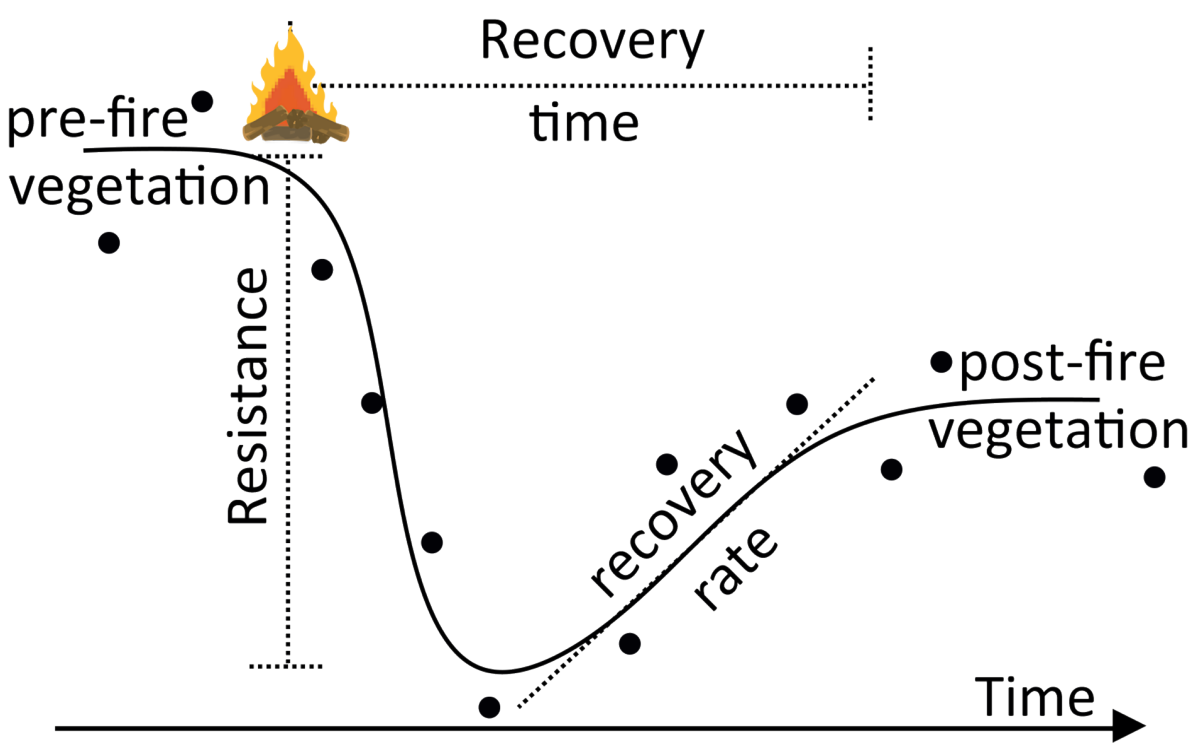

Figure 1: Example of resilience components estimated from pollen data (black dots).

for univariate satellite data, to a large spatial network of paleoecological records.

Finally, a third session aimed to predict which functional traits (i.e. morphological, physiological or phenological characteristics that influence how organisms respond to the environment; Diaz et al. 2013) would be responsible for ecosystems with high resistance, and which traits would be related to fast recovery rates. From a paleoecological perspective, it was clear that the key challenge is to develop tools which enable one to move from pollen assemblages (with their known biases related to taxonomic resolution and representation) to species-based trait databases derived from present-day ecological understanding and sampling (e.g. the TRY database; Kattge et al. 2011). This would then allow predictions made by ecological trait theory to be tested using paleoecological data. A workshop which aims to tackle these issues is planned in Utah, USA, in 2018.

During the workshop, a number of participants gave talks on their own methods to identify alternative stable states and early warning indicators in long-term ecological data. A critical theme that ran throughout the discussions was the need to have an underlying model to support the assumptions of resilience theory, since abrupt changes are not necessarily always associated with fold bifurcations (e.g. Williams et al. 2011). During the EcoRe 3 working group meeting, we distributed work tasks amongst members of the group, identified milestones for papers, and established the topics, timings and locations of future meetings. The spectacular Finse Alpine Research Station meant that many participants were able to try their hand at cross-country skiing as an antidote to the intense discussions over the course of the week. Further details of EcoRe 3 activities will be published on the website (www.pastglobalchanges.org/ini/wg/ecore3) and on social media outlets (see below)

- EcoRe3 on Facebook (visit page and request to join): https://www.facebook.com/ groups/286999515057710/

\section{-EcoRe3 on Twitter: @Eco_Re3}

- EcoRe3 on ResearchGate: https:// www.researchgate.net/project/EcoRe3Resistance-Recovery-and-Resilience-inLong-term-Ecological-Systems-2

\section{AFFILIATIONS}

'Department of Biology, University of Bergen, Norway 2Rezatec Ltd, Harwell, UK

${ }^{3}$ Department of Zoology, University of Oxford, UK ${ }^{4}$ School of Geography, University of Melbourne, Australia

${ }^{5}$ Department of Geography, University of Utah, Salt Lake City, USA

${ }^{6}$ Royal Botanic Gardens, Kew, London, UK

CONTACT

Alistair Seddon: Alistair.Seddon@uib.no

REFERENCES

Díaz S et al. (2013) Ecol Evol 3: 2958-2975

Kattge J et al. (2011) Glob Change Biol 17: 2905-2935

Williams JW et al. (2011) J Ecol 99: 664-677 